\title{
Effective conductivity and average polarizability of random polycrystals
}

\author{
Marco Avellaneda and Oscar Brunoa) \\ Courant Institute of Mathematical Sciences, 251 Mercer Street, New York, New York 10012
}

(Received 2 October 1989; accepted for publication 28 March 1990)

\begin{abstract}
A third-order expansion for the effective thermal conductivity tensor $\mathrm{K}^{*}$ of anisotropic polycrystalline cell materials is derived. The coefficients of the expansion are given in terms of the average polarizability tensor, a nondimensional quantity determined from the grain shape and crystallographic orientation distributions independent of other details of the microgeometry such as two (or more) particle correlation functions. Explicit numerical results for a wide variety of microgeometries made of ellipsoidal cells are obtained. This calculation uses a new method that exploits the symmetry properties of the effective conductivity tensor of a cell material as a function of the single-crystal conductivities.
\end{abstract}

\section{INTRODUCTION}

Single-phase polycrystals are assemblages of homogeneous grains or crystallites with arbitrary shapes, packed together so as to fill all space. The three principal thermal conductivities are the same in all grains, while the crystallographic orientations may vary randomly from one grain to another. A wide variety of crystalline solids, including most metals, are commonly found in polycrystalline form. According to Fourier's law of heat conduction, the temperature $T(x)$ and heat flux density $q(x)$ satisfy

$$
q(x)=-\mathrm{K}(x) \cdot \nabla T(x)
$$

and

$$
\nabla \cdot q(x)=0,
$$

where $K(x)$ is the local thermal conductivity tensor. We can write this tensor in the form

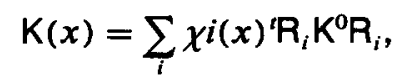

where $\chi_{i}(x)=1$ if $x$ is in grain $i$ and $\chi_{i}(x)=0$ otherwise;

$$
\mathrm{K}^{(0)}=\operatorname{diag}\left(k_{1}, k_{2}, k_{3}\right) \text {, }
$$

where $k_{j}, 1 \leqslant j \leqslant 3$ are the principal conductivities of the crystalline phase and ${ }^{t} \mathrm{R}_{i} \mathrm{~K}^{(0)} \mathrm{R}_{i}$ is the conductivity in grain $i$, $i=1,2,3, \ldots$, where $R_{i}$ are suitable rotations. In (2), it is implicity understood that the normal component of $q(x)$ is continuous across grain boundaries. We assume for simplicity that the medium occupies all space and that $K(x)$ is ergodic. Solutions of (1) and (2) can then be decomposed into the average and fluctuating components

$$
\begin{aligned}
& \nabla T(x)=\langle\nabla T\rangle+(\nabla T)^{\prime}(x), \\
& \mathbf{q}(x)=\langle\mathbf{q}\rangle+\mathbf{q}^{\prime}(x) .
\end{aligned}
$$

The effective conductivity tensor $K^{*}$ is defined by the relation

$$
\langle\mathbf{q}\rangle=-\mathrm{K}^{*} \cdot\langle\nabla T\rangle
$$

as $\langle\nabla T\rangle$ varies over all three-vectors. Physcially, $\mathrm{K}^{*}$ represents the conductivity of an equivalent homogeneous material and can be determined experimentally by direct or indirect measurements on samples which are large compared to the

\footnotetext{
a) Present address: School of Mathematics, University of Minnesota, Minneapolis, MN 55455.
}

typical grain size. ${ }^{1}$

The theoretical determination of $K^{*}$ is made difficult by the complex character of Eqs. (1) and (2) for realistic microgeometries. Also, for real materials, only limited information is available on the microgeometry, usually through measurements of correlation functions or the grain shape distribution. Considerable work has been done to estimate $\mathrm{K}^{*}$ starting with Voigt, ${ }^{2}$ Reuss, ${ }^{3}$ and Wiener, ${ }^{4}$ who derived upper and lower bounds on the effective conductivity $k^{*}$ of isotropic aggregates:

$$
\begin{aligned}
k_{R} & \equiv 3 /\left(1 / k_{1}+1 / k_{2}+1 / k_{3}\right) \leqslant k^{*} \leqslant k_{V} \\
& \equiv \frac{1}{3}\left(k_{1}+k_{2}+k_{3}\right) .
\end{aligned}
$$

Recently, this interval was narrowed to

$$
k_{s} \leqslant k^{*} \leqslant k_{V} \text {, }
$$

where $k_{S}$ is the positive root of

$$
k_{s}^{3}+\left(k_{1}+k_{2}+k_{3}\right) k_{s}^{2}-4 k_{1} k_{2} k_{3}=0
$$

and the new interval was shown to be, in a sense, optimal. ${ }^{5}$ However, the size of the interval (8) is too wide to reasonably determine $k^{*}$ even for moderately anisotropic crystals. More efficient estimates on the effective conductivity that incorporate additional statistical information were derived in 1969 and 1970 by Molyneux, ${ }^{6,7}$ who obtained a perturbation expansion for $k^{*}$ valid to third order in $\delta k_{i}=k_{i}-1$, $1 \leqslant i \leqslant 3$, and related bounds. This expansion incorporates the two- and three-point correlation functions of the material. The statistical information needed as input in such expressions must then be determined experimentally, e.g., by image processing techniques. ${ }^{8,9}$

We will focus here on a statistical model introduced by Miller, ${ }^{10}$ for which there exist estimates on $k^{*}$ which are significantly narrower than the $k_{s}-k_{V}$ bounds and yet more explicit than the Molyneux bounds. These estimates require as input the particle shape distribution and crystallographic orientation distribution within grains. Specifically, we will assume that (i) the crystallographic orientations of different grains are statistically independent and (ii) the crystallographic orientation within each grain is distributed randomly in such a way that for any rotation $R$, the six conductivities

$$
{ }^{t} \mathrm{R} \cdot \operatorname{diag}\left(k_{i}, k_{j}, k_{l}\right) \cdot \mathrm{R},
$$

obtained by permutation of the indices $i, j, k$, arise with equal 
probability. Assumption (ii) is satisfied, for instance, if the crystallographic orientation is uniformly distributed (and then all orientations are equally likely). Such assumptions determine the correlation functions to some extent, but not completely. For instance, the correlation length is of the order of one particle diameter. For isotropic aggregates of this type, Hashin and Shtrikman ${ }^{11}$ and Walpole ${ }^{12}$ showed that

$$
k^{*}=k_{V}-\frac{1}{9} \sum_{i=1}^{3}\left(k_{i}-k_{V}\right)^{2}+o(\delta k)^{2}
$$

and

$$
\begin{aligned}
& k_{1} \frac{4 k_{1}^{2}+8 k_{1} k_{2}+8 k_{1} k_{3}+7 k_{2} k_{3}}{16 k_{1}^{2}+5 k_{1} k_{2}+5 k_{1} k_{3}+k_{2} k_{3}} \\
& \quad \leqslant k^{*} \leqslant k_{3} \frac{4 k_{3}^{2}+8 k_{3} k_{2}+8 k_{1} k_{3}+7 k_{1} k_{2}}{16 k_{3}^{2}+5 k_{3} k_{2}+5 k_{3} k_{1}+k_{1} k_{2}}
\end{aligned}
$$

if $k_{1} \leqslant k_{2} \leqslant k_{3}$.

Subsequently, Willemse and Caspers ${ }^{13}$ calculated the corresponding third-order expansion for $k^{*}$ by assuming uniformly distributed crystallographic orientations. This expansion depends on a single parameter related to the particle shape distribution.

In this paper, we introduce a new approach for estimating the effective conductivity which fully exploits the symmetry properties implied by the statistical assumptions (i) and (ii). Our main result is a third-order Taylor expansion of the anisotropic tensor $\mathrm{K}^{*}\left(k_{1}, k_{2}, k_{3}\right)$ in $\delta k_{i}$. The coefficients of the expansion depend on the average polarizability tensor of the aggregate, a quantity determined only from the grain shape and crystallographic orientation distributions and independent from other details of the microgeometry. We recover the results of Hashin and Shtrikman, ${ }^{11}$ Walpole, ${ }^{12}$ and Willemse and Caspers ${ }^{13}$ as a special case when $K^{*}$ is isotropic (see Appendix B).

\section{THE MAIN RESULT: RELATION BETWEEN CONDUCTIVITY AND AVERAGE POLARIZABILITY}

Consider the Taylor series expansion of $\mathrm{K}^{*}=\mathrm{K}^{*}\left(k_{1}, k_{2}, k_{3}\right)$ near $k_{i}=1$ :

$$
\begin{aligned}
& \mathrm{K}^{*}\left(k_{1}, k_{2}, k_{3}\right)= \mathrm{I} \\
&+\sum_{i=1}^{3} \frac{\partial}{\partial k_{i}} \mathrm{~K}^{*}(1,1,1)\left(\delta k_{i}\right) \\
&+\frac{1}{2} \sum_{i, j=1}^{3} \frac{\partial^{2}}{\partial k_{i} \partial k_{j}} \mathrm{~K}^{*}(1,1,1)\left(\delta k_{i}\right)\left(\delta k_{j}\right) \\
&+\frac{1}{6} \sum_{i, j=1}^{3} \frac{\partial^{3}}{\partial k_{i} \partial k_{j} \partial k_{l}} \mathrm{~K}^{*}(1,1,1)\left(\delta k_{i}\right) \\
& \times\left(\delta k_{j}\right)\left(\delta k_{l}\right)+\cdots .
\end{aligned}
$$

From first principles,

$$
\mathrm{K}^{*}\left(\lambda k_{1}, \lambda k_{2}, \lambda k_{3}\right)=\lambda \mathrm{K}^{*}\left(k_{1}, k_{2}, k_{3}\right) .
$$

Also, we have

$$
\mathrm{K}^{*}\left(k_{1}, k_{2}, k_{3}\right)=\mathrm{K}^{*}\left(k_{i}, k_{j}, k_{l}\right), \quad i \neq j \neq l
$$

since our statistical assumptions ensure that $k_{1}, k_{2}, k_{3}$ are interchangeable. Differentiating $\mathrm{K}^{*}$ at $k_{i}=1$ and using (14) and (15), we obtain the relations

$$
\begin{aligned}
\frac{\partial}{\partial k_{i}} K^{*}(1,1,1) & =\frac{1}{3} \mathrm{I}, \quad 1 \leqslant i \leqslant 3, \\
\frac{\partial^{2}}{\partial k_{1}^{2}} \mathrm{~K}^{*}(1,1,1) & =\frac{\partial^{2}}{\partial k_{2}^{2}} \mathrm{~K}^{*}(1,1,1)=\frac{\partial^{2}}{\partial k_{3}^{2}} \mathrm{~K}^{*}(1,1,1) \\
& \equiv \mathrm{A},
\end{aligned}
$$

$$
\frac{\partial^{2}}{\partial k_{i} \partial k_{j}} \mathrm{~K}^{*}(1,1,1)=-\frac{1}{2} \frac{\partial^{2}}{\partial k_{1}^{2}} \mathrm{~K}^{*}(1,1,1), \quad i \neq j,
$$$$
\frac{\partial^{2}}{\partial k_{1}^{3}} \mathrm{~K}^{*}(1,1,1)=\frac{\partial^{3}}{\partial k_{2}^{3}} \mathrm{~K}^{*}(1,1,1)=\frac{\partial^{3}}{\partial k_{3}^{3}} \mathrm{~K}^{*}(1,1,1)
$$

$$
\begin{aligned}
\frac{\partial^{3}}{\partial k_{i}^{2} \partial k_{j}} \mathrm{~K}^{*}(1,1,1)= & -\frac{1}{2} \frac{\partial^{3}}{\partial k_{1}^{3}} \mathrm{~K}^{*}(1,1,1) \\
& -\frac{1}{2} \frac{\partial^{2}}{\partial k_{1}^{2}} \mathrm{~K}^{*}(1,1,1), \quad i \neq j,
\end{aligned}
$$

and

$$
\begin{aligned}
\frac{\partial^{3}}{\partial k_{1} \partial k_{2} \partial k_{3}} \mathrm{~K}^{*}(1,1,1)= & \frac{\partial^{3}}{\partial k_{1}^{3}} \mathrm{~K}^{*}(1,1,1) \\
& +\frac{3}{2} \frac{\partial^{2}}{\partial k_{1}^{2}} \mathrm{~K}^{*}(1,1,1) .
\end{aligned}
$$

Equation (20) was obtained by differentiating the relation $\mathrm{K}^{*}\left(k_{1}, k_{1}, 1\right)=k_{1} \mathrm{~K}^{*}\left(1,1, k_{1}^{-1}\right)$ and using (19), while Eq. (21) was obtained using the identity $\mathrm{K}^{*}\left(k_{1}, k_{2}, k_{3}\right)=k_{3} \mathrm{~K}^{*}\left(k_{1} / k_{3}, k_{2} / k_{3}, 1\right)$ together with (19) and (20). Thus we see that the third-order Taylor expansion (13) depends only on the two tensors of microgeometric parameters $A$ and $B$. For later use in comparison with other work, we observe that this expansion can be rewritten in the form

$$
\begin{aligned}
K^{*}\left(k_{1}, k_{2}, k_{3}\right)= & k_{V}\left[1+\frac{3}{4} \mathrm{~A} \sum_{i=1}^{3}\left(\frac{k_{i}}{k_{V}}-1\right)^{2}\right. \\
& \left.+\left(\frac{3}{4} \mathrm{~A}+\frac{3}{4} \mathrm{~B}\right) \sum_{i=1}^{3}\left(\frac{k_{i}}{k_{V}}-1\right)^{3}\right] \\
& +o\left(\left|\frac{k_{i}}{k_{V}}-1\right|\right)^{3}
\end{aligned}
$$

with $k_{V}=\frac{1}{3}\left(k_{1}+k_{2}+k_{3}\right)$. To further determine the tensors $A$ and $B$, we introduce the averge polarizability tensor. Consider first a configuration consisting of a single grain $G$ with the conductivity $\mathrm{K}_{G}={ }^{t} \mathrm{RK} \mathrm{K}^{(0)} \mathrm{R}$ embedded in an isotropic medium with unit conductivity. Given an arbitrary threevector e, denote by $T_{\mathrm{e}}^{G}(x)$ the temperature field which solves the corresponding field equations and such that 
$\lim _{|x| \rightarrow \infty} \nabla T_{e}^{G}(x)=$ e. The single-grain polarizability tensor $\mathscr{L}_{G}$ is defined by the relation

$$
{ }^{t} \mathrm{e} \cdot \mathscr{L}_{G} \cdot \mathrm{e}=\frac{1}{\operatorname{Vol}(G)} \int_{G} \nabla T_{\mathrm{e}}^{G}(x) \cdot\left(\mathrm{K}_{G}-I\right) \cdot \mathrm{e} d x
$$

The average polarizability tensor of the polycrystal is defined by

$$
\mathscr{L}=\left\langle\mathscr{L}_{G}\right\rangle,
$$

where the average is taken over the grain shape and crystallographic orientation distributions. Physcially, $\mathscr{L}$ represents the first-order correction to the value of the effective conductivity of an isotropic medium of unit conductivity containing an embedded dilute array of grains randomly sampled from the polycrystal (see Fig. 1). This tensor can be explicitly computed for several microgeometries (see Sec. III).

The identities relating A, B, and the average polarization tensor $\mathscr{L}$ for general grain shapes and orientations are

$$
\mathrm{A}=\frac{2}{3}\left[\frac{\partial^{2}}{\partial k_{1}^{2}} \mathscr{L}(1,1,1)-\frac{\partial^{2}}{\partial k_{1} \partial k_{2}} \mathscr{L}(1,1,1)\right]
$$

and

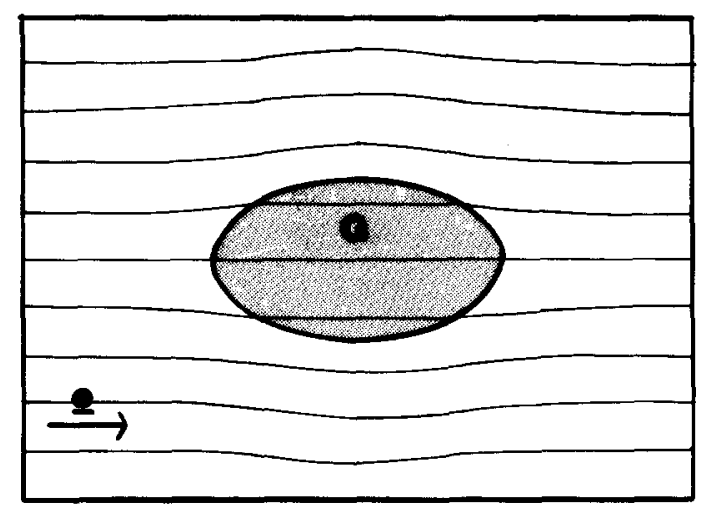

(a)

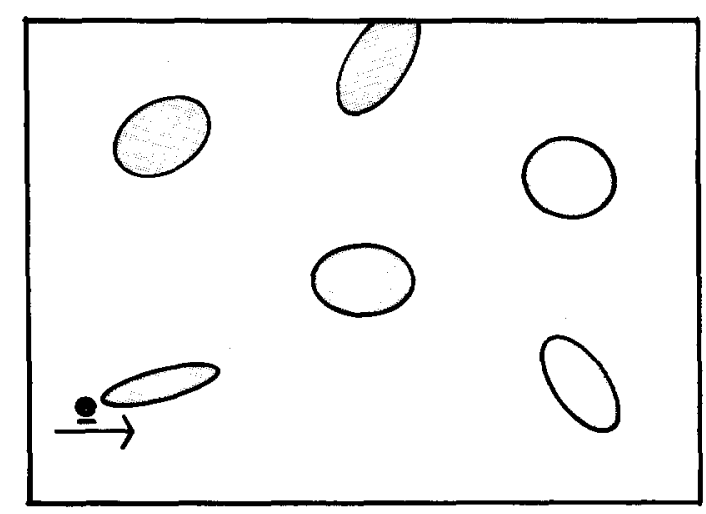

(b)

FIG. 1. (a) A schematic view of the single-grain configuration and the corresponding field lines of $\nabla T_{\text {. }}^{G}$. The conductivity is equal to $K_{G}$ inside the grain and $I$ outside. (b) The average polarizability $\mathscr{L}=\left\langle\mathscr{L}_{G}\right\rangle$ satisfies $\mathrm{K}_{\mathrm{efr}}^{*}=1+f \mathscr{L}+o(N)$, where $\mathrm{K}_{\mathrm{eff}}^{*}$ is the effective conductivity of a configuration consisting of a dilute array of randomly selected grains occupying a volume fraction $f$ and surrounded by a medium with the conductivity $\mathrm{K}=\mathrm{I}$.

$$
\begin{aligned}
\mathrm{B}= & \frac{2}{9} \frac{\partial^{3}}{\partial k_{1}^{3}} \mathscr{L}(1,1,1)-\frac{2}{3} \frac{\partial^{3}}{\partial k_{1}^{2} \partial k_{2}} \mathscr{L}(1,1,1) \\
& +\frac{4}{9} \frac{\partial^{3}}{\partial k_{1} \partial k_{2} \partial k_{3}} \mathscr{L}(1,1,1) \\
& -\frac{2}{3} \frac{\partial^{2}}{\partial k_{1}^{2}} \mathscr{L}(1,1,1)+\frac{2}{3} \frac{\partial^{2}}{\partial k_{1} \partial k_{2}} \mathscr{L}(1,1,1)
\end{aligned}
$$

Formulas (25) and (26) constitute the main new result of this paper: They are derived in Secs. IV-VI.

We point out that there exists a general linear relationship between the coefficients of the classical perturbation expansion in the conductivity contrast of a cell material and the expansion in powers of the volume fraction for the conductivity of a uniform reference medium containing a dilute dispersion of randomly selected cells from the composite. Such a relation was first systematically derived in Bruno's thesis, ${ }^{14}$ although some special cases of it already appeared in the work of Miller ${ }^{10}$ and Elsayed. ${ }^{15}$ The present results consist of an application of this general principle to singlephase polycrystals. It was shown that the computation of fourth-order and higher-order expansions requires the input of quantities related to the second- (and higher) order corrections to the conductivity of a uniform medium containing a dilute array of cells. ${ }^{14,15}$ Such corrections necessarily involve input of quantities related to interactions of two or more particles.

\section{AGGREGATES OF ELLIPSOIDAL GRAINS}

According to a classical result in potential theory going back to Maxwell, ${ }^{16}$ the field $\nabla T_{e}^{G}(x)$ is uniform within the grain $G$ if $G$ is an ellipsoid. This allows us to obtain an explicit formula for $\mathscr{L}_{G}$. Thus for aggregates consisting of ellipsoidal grains-not necessarily with the same shapes or orientations-the average polarizability $\mathscr{L}=\left\langle\mathscr{L}_{G}\right\rangle$ can be determined explicitly.

Fixing a Cartesian reference frame, $e^{(1)}, e^{(2)}, e^{(3)}$, the shape and position of a single ellipsoidal grain is determined by three unit vectors $n^{(1)}, n^{(2)}, n^{(3)}$ in the directions of its semiaxes and by the corresponding depolarization factors $L_{i}$, $1 \leqslant i \leqslant 3$, which satisfy $0 \leqslant L_{i} \leqslant 1, \Sigma_{i} L_{i}=1$. If the position of the vector $\mathbf{n}^{(i)}$ is specified by

$$
\mathbf{n}^{(i)}={ }^{i} \mathbf{e}^{(i)},
$$

where $O$ is a rotation matrix, and the conductivity within the grain is $K_{G}={ }^{t} R K^{(0)} R$, it can be shown that

$$
\mathscr{L}_{G}=\delta \mathrm{K}_{G}\left(I+{ }^{\mathrm{O}} \mathrm{OLO} \delta \mathrm{K}_{G}\right)^{-1},
$$

where

$$
\delta \mathrm{K}_{G}={ }^{t} \mathrm{R}\left(\mathrm{K}^{(0)}-\mathrm{I}\right) \mathrm{R}={ }^{t} \mathrm{R} \delta \mathrm{K}^{(0)} \mathrm{R}
$$

and

$$
L=\operatorname{diag}\left(L_{1}, L_{2}, L_{3}\right) .
$$

Thus denoting by $d \mu(L, O, R)$ the joint grain shape-crystallographic distribution orientation, we have, from (24) and (28), 
$\mathscr{L}=\int{ }^{t} \mathrm{R} \delta \mathrm{K}^{(0)} \mathrm{R}\left(\mathrm{I}+{ }^{\circ} \mathrm{OLO} \mathrm{L}^{\prime} \mathrm{R} \delta \mathrm{K}^{(0)} \mathrm{R}\right)^{-1} d \mu(\mathrm{L}, \mathrm{O}, \mathrm{R})$.

Computing the second and third partial derivatives of $\mathscr{L}$ at $k_{i}=1$ and substituting their expressions into (25) and (26), we obtain an explicit third-order expansion for aggregates of ellipsoidal grains satisfying assumptions (i) and (ii). This calculation is straightforward, although rather lengthy (see Appendix C). When the orientation distribution within each grain is uniformly distributed, the corresponding expressions for $A$ and $B$ are

$$
A=-\frac{2}{45} \int \text { OLO } d \mu-\frac{2}{15} \text { I }
$$

and

$$
\begin{aligned}
\mathrm{B}= & \frac{32}{315} \int \mathrm{O}^{\mathrm{O}} \mathrm{L}^{2} \mathrm{O} d \mu-\frac{2}{105} \int \operatorname{tr} \mathrm{L}^{2} \mathrm{~d} \mu \\
& +\frac{2}{315} \int \mathrm{O} \mathrm{O} \mathrm{O} d \mu-\frac{4}{21} \mathrm{l} .
\end{aligned}
$$

For instance, if the grains are oriented uniformly in all directions,

$$
A=-(4 / 27) \mid
$$

and

$$
\mathrm{B}=\left[\frac{2}{135}\left\langle L_{1}^{2}+L_{2}^{2}+L_{3}^{2}\right\rangle+\frac{26}{135}\right] \mathrm{l},
$$

where $\left\langle L_{1}^{2}+L_{2}^{2}+L_{3}^{2}\right\rangle$ denotes averaging over all grain shapes. For spherical grains, we obtain, with $L_{1}=L_{2}=L_{3}=\frac{1}{3}$,

$$
\mathrm{B}_{S}=\frac{16}{81} \text {. }
$$

In the limiting cases of randomly oriented lamellar particles ( $\left.L_{1}=1, \quad L_{2}=L_{3}=0\right)$ and needle-like particles $\left(L_{1}=L_{2}=\frac{1}{2}, L_{3}=0\right)$ we obtain, respectively,

$$
\mathrm{B}_{L}=\frac{28}{135} \mathrm{I}, \quad \mathrm{B}_{N}=\frac{53}{270} \mathrm{I} .
$$

\section{AN ASSOCIATED N-PHASE POLYCRYSTAL}

To incorporate into the calculation the statistical independence of the crystallographic orientations of different cells, we introduce a mathematical artifice. Let $N$ be a large positive integer. We assign randomly an integer between 1 and $N$ to each grain. In this way, we obtain $N$ statistically equivalent sets of grains, each one formed by those cells with the same assigned number. Next, we replace the single-crystal conductivity matrix $\mathrm{K}^{0}$ by

$$
\mathrm{K}^{(j)}=\operatorname{diag}\left(x_{j}, y_{j}, z_{j}\right)
$$

in all grains of the set with the assigned number $j, 1 \leqslant j \leqslant N$, where $\left(x_{j}, y_{j}, z_{j}\right)$ are $N$ triples of positive numbers. The local conductivity tensor of this $N$-phase polycrystal is

$$
\mathrm{K}_{N}(x)=\sum_{j=1}^{N}\left[\sum_{\substack{i \\ \text { grain } i \text { has assigned number } j}} \chi_{i}(x)^{t} \mathrm{R}_{i} \mathrm{~K}^{(j)} \mathrm{R}_{i}\right] .
$$

Let

$$
\mathrm{K}_{N}^{*}=\mathrm{K}_{N}^{*}\left[\left(x_{1}, y_{1}, z_{1}\right), \ldots,\left(x_{N}, y_{N}, z_{N}\right)\right]
$$

denote the corresponding effective conductivity, viewed as a function of the $3 N$ variables $\left(x_{i}, y_{i}, z_{i}\right), i \leqslant N$. Clearly, by construction,

$$
\begin{aligned}
\mathrm{K}_{N}^{*}\left[\left(x_{1}, y_{1}, z_{1}\right), \ldots,\left(x_{i}, y_{i}, z_{i}\right), \ldots,\left(x_{j}, y_{j}, z_{j}\right), \ldots,\left(x_{N}, y_{N}, z_{N}\right)\right] \\
\quad=\mathrm{K}^{*}\left[\left(x_{1}, y_{1}, z_{1}\right), \ldots,\left(x_{j}, y_{j}, z_{j}\right), \ldots,\left(x_{i}, y_{i}, z_{i}\right), \ldots,\left(x_{N}, y_{N}, z_{N}\right)\right]
\end{aligned}
$$

for all $i \neq j$. Moreover, by assumption (ii) on the crystallographic orientation distributions,

$$
\begin{aligned}
\mathrm{K}_{N}^{*}\left[\left(x_{1}, y_{1}, z_{1}\right), \ldots,\left(x_{i}, y_{i}, z_{i}\right), \ldots,\left(x_{N}, y_{N}, z_{N}\right)\right] & =\mathrm{K}_{N}^{*}\left[\left(x_{1}, y_{1}, z_{1}\right), \ldots,\left(y_{i}, x_{i}, z_{i}\right), \ldots,\left(x_{N}, y_{N}, z_{N}\right)\right], \\
& =\mathrm{K}_{N}^{*}\left[\left(x_{1}, y_{1}, z_{1}\right), \ldots,\left(x_{i}, z_{i}, y_{i}\right), \ldots,\left(x_{N}, y_{N}, z_{N}\right)\right], \text { etc. }
\end{aligned}
$$

The effective conductivity of the original single-phase polycrystal satisfies

$$
\mathrm{K}^{*}\left[\left(k_{1}, k_{2}, k_{3}\right)\right]=\mathrm{K}_{N}^{*}\left[\left(k_{1}, k_{2}, k_{3}\right), \ldots,\left(k_{1}, k_{2}, k_{3}\right), \ldots,\left(k_{1}, k_{2}, k_{3}\right)\right] .
$$

We shall assume first, for simplicity, that there are finitely many grains per unit volume. This assumption is removed in Appendix A. Under this condition, each of the $N$ phases is dilute. ${ }^{10}$ Hence

$$
\begin{aligned}
\mathrm{K}_{N}^{*} & {\left[\left(x_{1}, y_{1}, z_{1}\right),(1,1,1), \ldots,(1,1,1)\right] } \\
& =1+(1 / N) \mathscr{L}\left(x_{1}, y_{1}, z_{1}\right)+o(1 / N) .
\end{aligned}
$$

Equation (44) together with (43), provide a useful math- ematical relation between the average polarizability and the conductivity of the polycrystal, which we exploit in the next sections.

\section{EXPRESSION FOR A IN TERMS OF $\partial^{2} \mathscr{L}$}

We set

$$
\xi_{1}=\frac{\partial^{2}}{\partial x_{1}^{2}} K_{N}^{*}[(1,1,1), \ldots,(1,1,1)],
$$




$$
\xi_{2}=\frac{\partial^{2}}{\partial x_{1} \partial y_{1}} K_{N}^{*}[(1,1,1), \ldots,(1,1,1)],
$$

and

$$
\xi_{3}=\frac{\partial^{2}}{\partial x_{1} \partial x_{2}} K_{N}^{*}[(1,1,1), \ldots,(1,1,1)],
$$

Differentiating (43) twice at $k_{i}=1$, we obtain

$$
\mathrm{A}=\frac{\partial^{2}}{\partial k_{1}^{2}} \mathrm{~K}^{*}(1,1,1)=N \xi_{1}+N(N-1) \xi_{3}
$$

Three independent linear relations between $\xi_{1}, \xi_{2}, \xi_{3}$ and the second derivatives of $\mathscr{L}$ are obtained by differentiating the equations

$$
\begin{aligned}
& \mathrm{K}_{N}^{*}[(z, 1,1),(1,1,1), \ldots,(1,1,1)] \\
& \quad=1+(1 / N) \mathscr{L}(z, 1,1)+o(1 / N), \\
& \mathrm{K}_{N}^{*}[(z, z, z),(1,1,1), \ldots,(1,1,1)] \\
& \quad=1+(1 / N) \mathscr{L}(z, z, z)+o(1 / N),
\end{aligned}
$$

and

$$
\mathrm{K}_{N}^{*}[(z, z, z), \ldots,(z, z, z)]=z .
$$

We obtain, respectively,

$$
\begin{aligned}
\xi_{1}=\frac{1}{N} \frac{\partial^{2}}{\partial k_{1}^{2}} \mathscr{L}(1,1,1)+o\left(\frac{1}{N}\right) \\
3 \xi_{1}+6 \xi_{2}=\frac{3}{N}\left[\frac{\partial^{2}}{\partial k_{1}^{2}} \mathscr{L}(1,1,1)\right. \\
\left.+2 \frac{\partial^{2}}{\partial k_{1} \partial k_{2}} \mathscr{L}(1,1,1)\right]+o\left(\frac{1}{N}\right),
\end{aligned}
$$

and

$$
\xi_{1}+2 \xi_{2}+3(N-1) \xi_{3}=0,
$$

which yield

$$
\begin{aligned}
\xi_{1}= & \frac{1}{N} \frac{\partial^{2}}{\partial k_{1}^{2}} \mathscr{L}(1,1,1)+o\left(\frac{1}{N}\right), \\
\xi_{2}= & \frac{1}{N} \frac{\partial^{2}}{\partial k_{1} \partial k_{2}} \mathscr{L}(1,1,1)+o\left(\frac{1}{N}\right), \\
\xi_{3}= & -\frac{1}{3 N(N-1)}\left[\frac{\partial^{2}}{\partial k_{1}^{2}} \mathscr{L}(1,1,1)\right. \\
& \left.+2 \frac{\partial^{2}}{\partial k_{1} \partial k_{2}} \mathscr{L}(1,1,1)\right]+o\left(\frac{1}{N^{2}}\right) .
\end{aligned}
$$

$$
\begin{aligned}
& \eta_{1}=\frac{1}{N} \frac{\partial^{3}}{\partial k_{1}^{3}} \mathscr{L}(1,1,1)+o\left(\frac{1}{N}\right), \\
& 2 \eta_{1}+6 \eta_{2}=\frac{1}{N}\left[2 \frac{\partial^{3}}{\partial k_{1}^{3}} \mathscr{L}(1,1,1)+6 \frac{\partial^{3}}{\partial k_{1}^{2} \partial k_{2}} \mathscr{L}(1,1,1)\right]+o\left(\frac{1}{N}\right),
\end{aligned}
$$


$3 \eta+18 \eta_{2}+6 \eta_{3}=\frac{1}{N}\left[3 \frac{\partial^{3}}{\partial k_{1}^{3}} \mathscr{L}(1,1,1)+18 \frac{\partial^{3}}{\partial k_{1}^{2} \partial k_{2}} \mathscr{L}(1,1,1)+6 \frac{\partial^{3}}{\partial k_{1} \partial k_{2} \partial k_{3}} \mathscr{L}(1,1,1)\right]+o\left(\frac{1}{N}\right)$,

$3 N \eta_{1}+18 N \eta_{2}+6 N \eta_{3}+27 N(N-1) \eta_{4}+54 N(N-1) \eta_{5}+27 N(N-1)(N-2) \eta_{6}=0$,

$3 N \eta_{1}+18 N \eta_{2}+6 N \eta_{3}+27(N-1)(N-2) \eta_{4}+54(N-1)(N-2) \eta_{5}$

$+27(N-1)(N-2)(N-3) \eta_{6}+9(N-1) \xi_{1}+18(N-1) \xi_{2}+27(N-1)(N-2) \xi_{3}=0$,

and

$$
\begin{aligned}
3 N \eta_{1} & +6 N \eta_{2}+15 N(N-1) \eta_{4}+12 N(N-1) \eta_{5}+9 N(N-1)(N-2) \eta_{6} \\
& +6 N \xi_{1}+6 N \xi_{2}+12 N(N-1) \xi_{3}=0 .
\end{aligned}
$$

Equations (59)-(61) can be solved in terms of $\partial^{3} \mathscr{L}$ as

$$
\begin{aligned}
& \eta_{1}=\frac{1}{N} \frac{\partial^{3}}{\partial k_{1}^{3}} \mathscr{L}(1,1,1)+o\left(\frac{1}{N}\right), \\
& \eta_{2}=\frac{1}{N} \frac{\partial^{3}}{\partial k_{1}^{2} \partial k_{2}} \mathscr{L}(1,1,1)+o\left(\frac{1}{N}\right), \\
& \eta_{3}=\frac{1}{N} \frac{\partial^{3}}{\partial k_{1} \partial k_{2} \partial k_{3}} \mathscr{L}(1,1,1)+o\left(\frac{1}{N}\right) .
\end{aligned}
$$

Equations (62) $-(64)$ can be cast in the form

$$
\begin{aligned}
\eta_{4}+2 \eta_{5}+(N-2) \eta_{6} \\
=-[1 / 9(N-1)]\left(\eta_{1}+6 \eta_{2}+2 \eta_{3}\right),
\end{aligned}
$$

$$
\begin{aligned}
\boldsymbol{\eta}_{4}+ & 2 \eta_{5}+(N-3) \eta_{6} \\
& =-[N / 9(N-1)(N-2)]\left(\eta_{1}+6 \eta_{2}+2 \eta_{3}\right),
\end{aligned}
$$

and

$$
\begin{aligned}
5 \eta_{4}+ & 4 \eta_{5}+3(N-2) \eta_{6} \\
= & -[1 /(N-1)]\left(\eta_{1}+2 \eta_{2}\right)-[2 /(N-1)] \\
& \times\left[\xi_{1}+\xi_{2}+2(N-1) \xi_{3}\right],
\end{aligned}
$$

where the last equation in (52) was used to simplify the rhs of (67). Upon solving (66)-(68) in $\eta_{4}, \eta_{5}, \eta_{6}$ and using (65), we obtain

$$
\begin{aligned}
N(N-1) \eta_{4} & \\
= & -\frac{2}{9} \frac{\partial^{2}}{\partial k_{1}^{2}} \mathscr{L}(1,1,1)+\frac{2}{9} \frac{\partial^{2}}{\partial k_{1} \partial k_{2}} \mathscr{L}(1,1,1) \\
& -\frac{1}{3} \frac{\partial^{3}}{\partial k_{1}^{3}} \mathscr{L}(1,1,1)-\frac{2}{3} \frac{\partial^{3}}{\partial k_{1}^{2} \partial k_{2}} \mathscr{L}(1,1,1)+o(1),
\end{aligned}
$$

$$
\begin{aligned}
& N(N-1) \eta_{5} \\
&= \frac{1}{9} \frac{\partial^{2}}{\partial k_{1}^{2}} \mathscr{L}(1,1,1)-\frac{1}{9} \frac{\partial^{2}}{\partial k_{1} \partial k_{2}} \mathscr{L}(1,1,1) \\
&-\frac{2}{3} \frac{\partial^{3}}{\partial k_{1}^{2} \partial k_{2}} \mathscr{L}(1,1,1)-\frac{1}{3} \frac{\partial^{3}}{\partial k_{1} \partial k_{2} \partial k_{3}} \mathscr{L}(1,1,1)+o(1),
\end{aligned}
$$

and

$$
\begin{aligned}
N(N-1)(N-2) \eta_{6} \\
=\frac{2}{9} \frac{\partial^{3}}{\partial k_{1}^{3}} \mathscr{L}(1,1,1)+\frac{4}{3} \frac{\partial^{3}}{\partial k_{1}^{2} \partial k_{2}} \mathscr{L}(1,1,1) \\
\quad+\frac{4}{9} \frac{\partial^{3}}{\partial k_{1} \partial k_{2} \partial k_{3}} \mathscr{L}(1,1,1)+o(1) .
\end{aligned}
$$

The expression for $\mathrm{B}$ in terms of $\partial^{2} \mathscr{L}$ and $\partial^{3} \mathscr{L}$ is obtained by substituting (65), (69), and (71) into formula (55) and taking the limit as $N \rightarrow \infty$.

\section{ACKNOWLEDGMENTS}

We are grateful to Professor G. Milton and Dr. J. Berryman for useful discussions which helped improve this paper.

This research was partially supported by a National 
Science Foundation Grant No. NSF-DMS-8802739 and by the US Army Research Office through Grants Nos. ARODAAL-0389K0039 and ARO-DAAL-0388K0110.

\section{APPENDIX A: REMOVAL OF THE ASSUMPTION OF FINITELY MANY GRAINS PER UNIT VOLUME}

For an arbitrary grain $G$ of finite volume, we set

$$
T_{e}^{G}(x)=\mathbf{e} x+T^{\prime}(x),
$$

so that

$$
{ }^{t} \mathrm{e} \cdot \mathscr{L}_{G} \cdot \mathrm{e}=\frac{1}{\operatorname{Vol}(G)} \int_{G} \nabla T^{\prime}(x) \cdot \delta \mathrm{K}_{G} \cdot \mathrm{e}+{ }^{t} \mathrm{e} \cdot \delta \mathrm{K}_{G} \cdot \mathrm{e} .
$$

The function $T^{\prime}(x)$ satisfies

$$
\nabla \cdot \mathrm{K}(x) \cdot \nabla T^{\prime}(x)+\nabla \cdot \chi_{G}(x) \cdot \delta \mathrm{K}_{G} \cdot \mathrm{e}=0,
$$

where $\chi_{G}$ is the characteristic function of $G$ and $\mathrm{K}(x)=\mathrm{K}_{G} \chi_{G}(x)+1\left(1-\chi_{G}(x)\right)$. Multiplying both sides of Eq. (A3) by $T^{\prime}(x)$ and integrating, we obtain

$$
\int_{G} \nabla T^{\prime}(x) \cdot \delta \mathrm{K}_{G} \cdot \mathrm{e} d x=-\int \nabla T^{\prime}(x) \cdot \mathrm{K}(x) \cdot \nabla T^{\prime}(x) d x .
$$

By the Cauchy-Schwartz inequality applied to (A4),

$$
\left(\int\left|\nabla T^{\prime}(x)\right|^{2} d x\right)^{1 / 2} \leqslant \frac{\operatorname{Vol}(G)^{1 / 2}}{\min \left(k_{1}, 1\right)}\left|\delta \mathrm{K}_{G} \cdot \mathbf{e}\right| .
$$

Hence, 'e $\mathscr{L}_{G} \cdot$ e can be estimated as follows:

$$
\begin{aligned}
\left|{ }^{t} \mathrm{e} \cdot \mathscr{L}_{G} \cdot \mathrm{e}\right| \leqslant & \frac{1}{\operatorname{Vol}(G)}\left(\int\left|\nabla T^{\prime}(x)\right|^{2} d x\right)^{1 / 2} \\
& \cdot \operatorname{Vol}(G)^{1 / 2}\left|\delta \mathrm{K}_{G} \mathrm{e}\right|+{ }^{t} \mathrm{e} \cdot \delta \mathrm{K}_{G} \cdot \mathrm{e} \\
\leqslant & \frac{1}{\min \left(k_{1}, 1\right)}\left|\delta \mathrm{K}_{G} \mathrm{e}\right|^{2}+{ }^{t} \mathrm{e} \cdot \delta \mathrm{K}_{G} \cdot \mathrm{e} .
\end{aligned}
$$

Equation (A6) implies that for $\left|\delta k_{i}\right|<\frac{1}{2}$, any entry of the tensor $\mathscr{L}_{G}$ satisfies

$$
\left|\mathscr{L}_{G}^{\mathrm{pq}}\left(k_{1}, k_{2}, k_{3}\right)\right| \leqslant 1 / 4 \min \left[k_{1}, 1\right]+\frac{1}{2} \leqslant 1, \quad 1 \leqslant p, q \leqslant 3 .
$$

Thus the polarizability tensor of a grain of conductivities $k_{i}$, $1 \leqslant i \leqslant 3$ with $\left|\delta k_{i}\right|<\frac{1}{2}$ is uniformly bounded, independent of the shape of the grain. Since $\mathscr{L}_{G}$ is an analytic function of $k_{1}$, $k_{2}, k_{3}$ [because $T^{\prime}$ satisfies the elliptic equation (A3)], similar uniform shape-independent bounds hold for all the derivatives of $\mathscr{L}_{G}$ at $k_{i}=1$.

Using this fact, we now remove the assumption that the polycrystal have finitely many grains per unit volume. Given an arbitrary grain configuration, we can construct a modified polycrystal by removing all grains of maximum diameter $\leqslant \epsilon$ (where $\epsilon$ is a small number) and replacing the resulting free space by new grains, so that the new aggregate has finitely many grains per unit volume. Note that the volume fraction of material in the original configuration, which has been removed and subsequently filled with new grains, tends to zero as $\epsilon \rightarrow 0$. Denote by $\mathscr{L}$ and $\widetilde{L}$ the average polarizability tensors corresponding to the original and modified configurations, respectively. The tensors $\mathscr{L}, \widetilde{L}$, and their derivatives are averages of the corresponding single-grain quantities, $\mathscr{L}_{G}$, and their derivatives over the grain shape and crystallographic orientation distributions. We conclude from the above analysis that

$$
\begin{gathered}
\max _{|\alpha|<3}\left\|D_{k}^{\alpha} \mathscr{L}(1,1,1)-D_{k}^{\alpha} \tilde{\mathscr{L}}(1,1,1)\right\| \\
\quad \leqslant C_{1} \times \text { (volume removed), }
\end{gathered}
$$

where $C_{1}$ is a numerical constant.

On the other hand, the conductivity tensor $\mathrm{K}^{*}\left(k_{1}, k_{2}, k_{3}\right)$ is known to be analytic in $k_{1}, k_{2}, k_{3}$ at $k_{i}=1$. Also, denoting the conductivity of the modified configuration by $\widetilde{K}^{*}$, it is known that

$$
\left\|\mathrm{K}^{*}-\widetilde{\mathrm{K}}^{*}\right\| \leqslant C_{2} \text { (volume removed) }{ }^{\nu},
$$

where $C_{2}$ and $v$ are positive constants which depend on $\max _{i} k_{i}$ and $\min _{i} k_{i}{ }^{17}{ }^{17}$ From this estimate and the analyticity of $K^{*}$ and $\widetilde{K}^{*}$, we conclude that

$$
\max _{|\alpha|<3}\left\|D_{k}^{\alpha} \mathrm{K}^{*}(1,1,1)-D_{k}^{\alpha} \widetilde{\mathrm{K}}^{*}(1,1,1)\right\|
$$

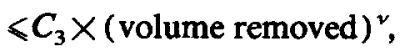

where $C_{3}$ is a numerical constant. Since the modified configuration has only finitely many grains per unit volume, the basic identies (25) and (26) relating $\partial^{2} \widetilde{\mathrm{K}}^{*}$ and $\partial^{3} \widetilde{\mathrm{K}}^{*}$ to the derivatives of $\mathscr{L}$ are now valid. To extend such identities to the conductivity and polarizability of the original configuration, we use the estimates (A8) and (A10). Accordingly,

$$
\begin{aligned}
& \mid \mathrm{A}- \frac{2}{3}\left[\frac{\partial^{2}}{\partial k_{1}^{2}} \mathscr{L}(1,1,1)-\frac{\partial^{2}}{\partial k_{1} \partial k_{2}} \mathscr{L}(1,1,1)\right] \mid \\
& \leqslant|\mathrm{A}-\widetilde{\mathrm{A}}|+\frac{2}{3}\left|\frac{\partial^{2}}{\partial k_{1}^{2}} \mathscr{L}(1,1,1)-\frac{\partial^{2}}{\partial k_{1}^{2}} \mathscr{L}(1,1,1)\right| \\
& \quad+\frac{2}{3}\left|\frac{\partial^{2}}{\partial k_{1} \partial k_{2}} \mathscr{L}(1,1,1)-\frac{\partial^{2}}{\partial k_{1} \partial k_{2}} \widetilde{\mathscr{L}}(1,1,1)\right| \\
& \leqslant C_{3} \text { (volume removed) }{ }^{\nu}+\frac{4 C_{1}}{3} \text { (volume removed). }
\end{aligned}
$$

Letting $\epsilon$ tend to zero, the volume removed tends to zero and we recover identity (25) for an arbitrary configuration. The approximation argument for $B$ is entirely similar.

\section{APPENDIX B: CALCULATION OF TRACE A AND APPLICATION TO ISOTROPIC POLYCRYSTALS}

The partial derivatives of the single-grain polarizability $\mathscr{L}_{G}\left(k_{1}, k_{2}, k_{3}\right)$ of an arbitrary bounded grain $G$ can be computed formally by differentiating both sides of (A2). This involves the differentiation of the function $T^{\prime}(x)$ in the parameters $k_{i}$ at $k_{i}=1,1 \leqslant i \leqslant 3$. In this appendix we show how such a calculation leads to the fact that tr $A$ is independent of the grain shape and orientation distributions. We set

$$
T_{i}(x)=\left.\frac{\partial}{\partial k_{i}}\right|_{\mathrm{k}=(1,1,1)} T^{\prime}(x)
$$


and note that differentiating Eq. (A3) once at $k_{i}=1$, this function satisfies

$$
\Delta T_{i}+\left({ }^{i} \mathbf{n}_{i} \cdot \mathbf{e}\right) \nabla \cdot \chi_{G} \mathbf{n}_{i}=0, \quad 1<i \leqslant 3,
$$

where $\mathbf{n}$ is a unit vector in the direction of the principal conductivity $k_{i}$. On the other hand, differentiating (A2) twice at $k_{i}=1$, one obtains

$$
\begin{aligned}
t e \cdot \frac{\partial^{2}}{\partial k_{i} \partial k_{j}} \tilde{L}(1,1,1) \cdot \mathrm{e} \\
=\frac{1}{\operatorname{Vol}(G)} \int_{G}\left(\nabla T_{i}(x) \cdot n_{j}\right)\left({ }^{\prime} n_{j} \cdot \mathbf{e}\right) \\
\quad+\frac{1}{\operatorname{Vol}(G)} \int_{G}\left(\nabla T_{j}(x) \cdot n_{i}\right)\left({ }^{\prime} \mathbf{n}_{i} \cdot \mathbf{e}\right) .
\end{aligned}
$$

We deduce from (B2) that formally,

$$
\nabla T_{i}(x)=\int_{\mathbf{R}^{3}} \Gamma(x-y) \cdot \mathbf{n}_{i}\left({ }^{t} \mathbf{n}_{i} \cdot \mathbf{e}\right) \chi_{\mathbf{G}}(y) d y,
$$

where

$$
\Gamma_{p q}(x)=\frac{\partial^{2}}{\partial x_{p} \partial x_{q}} \frac{1}{4 \pi|x|}=\frac{1}{4 \pi|x|^{3}}\left(\frac{3 x_{i} x_{j}}{|x|^{2}}-\delta_{i j}\right)
$$

is the kernel of the integral operator $\left(\partial^{2} / \partial x^{p} \partial x^{q}\right)(-\Delta)^{-1}$. The Fourier transform of $\Gamma(x)$ is

$$
\hat{\Gamma}_{p q}(\xi)=\int_{\mathbf{R}^{3}} \Gamma_{p q}(x) e^{i x \xi} d x=-\frac{\xi_{p} \xi_{q}}{|\xi|^{2}} .
$$

From (B4), we conclude that the Fourier transform of $\nabla T_{i}(x)$ is

$$
\nabla T_{i}(\xi)=-\left[\left({ }^{t} n_{i} \cdot e\right)\left({ }^{t} \xi \cdot n_{i}\right) \hat{\chi}_{G}(\xi) /|\xi|^{2}\right] \xi .
$$

Substituting expression (B5) into (B3) and using the Plancherel identity, we obtain

$$
\begin{aligned}
\text { tee } \frac{\partial^{2}}{\partial k_{i} \partial k_{j}} \mathscr{L}_{G}(1,1,1) \cdot \mathrm{e} \\
=-\frac{2}{\operatorname{Vol}(G)} \int_{\mathbf{R}^{3}} \frac{\left({ }^{t} \mathbf{n}_{i} \cdot \mathbf{e}\right)\left({ }^{t} \mathbf{n}_{j} \cdot \mathbf{e}\right)\left({ }^{t} \mathbf{n}_{i} \cdot \xi\right)\left({ }^{t} \mathbf{n}_{j} \cdot \xi\right)}{|\xi|^{2}} \\
\quad \times\left|\hat{\chi}_{G}(\xi)\right|^{2} d \xi .
\end{aligned}
$$

The trace of $\partial^{2} / \partial k_{i} \partial k_{j} \mathscr{L}_{G}(1,1,1)$ is computed by varying e over an orthonormal basis in (B6) and summing. Accordingly,

$$
\operatorname{tr} \frac{\partial^{2}}{\partial k_{i} \partial k_{j}} \mathscr{L}_{G}(1,1,1)=0, \quad \text { if } i \neq j
$$

and

$$
\begin{aligned}
& \operatorname{tr} \frac{\partial^{2}}{\partial k_{i}^{2}} \mathscr{L}_{G}(1,1,1) \\
& \quad=-\frac{2}{3 \operatorname{Vol}(G)} \int_{\mathbf{R}^{3}} \frac{\left({ }^{t} n_{i} \cdot \xi\right)^{2}}{|\xi|^{2}}\left|\hat{\chi}_{G}(\xi)\right|^{2} d \xi .
\end{aligned}
$$

Since the three principal conductivities $k_{1}, k_{2}, k_{3}$ are interchangeable,

$$
\left\langle\left({ }^{t} n_{i} \cdot \xi\right)^{2} /|\xi|^{2}\right\rangle=\frac{1}{3}, \quad \xi \in \mathbb{R}^{3}, \quad 1 \leqslant i \leqslant 3,
$$

where the brackets indicate averaging over the orientation distribution of the conductivity in grain $G$. Hence, the average polarizability tensor $\mathscr{L}$ satisfies

$$
\begin{aligned}
\operatorname{tr} \frac{\partial^{2}}{\partial k_{i}^{2}} \mathscr{L}(1,1,1) & =-\left\langle\frac{2}{3 \operatorname{Vol}(G)} \int_{\mathbf{R}^{3}}\left|\hat{\chi}_{G}(\xi)\right|^{2} d \xi\right\rangle \\
& =-\left\langle\frac{2}{3} \frac{1}{\operatorname{Vol}(G)} \cdot \operatorname{Vol}(G)\right\rangle \\
& =-\frac{2}{3} .
\end{aligned}
$$

From the basic formula for (25) (for $A$ ) we conclude that

$$
\operatorname{tr} A=-\frac{4}{9} \text {. }
$$

Substituting this result in the Taylor expansion (22) [for $\left.\mathrm{K}^{*}\left(k_{1}, k_{2}, k_{3}\right)\right]$, we obtain

$$
\frac{1}{3} \operatorname{tr} K^{*}\left(k_{1}, k_{2}, k_{3}\right)=k_{V}\left[1-\frac{1}{9} \sum_{i=1}^{3}\left(\frac{k_{i}}{k_{V}}-1\right)^{2}\right]
$$

$$
+o\left(\delta k_{i}^{2}\right) \text {. }
$$

In particular, we recover (11) for isotropic configurations. A similar calculation, which we omit, yields an expression for the trace of $B$ in terms of multiple integrals depending on the average grain shape, which allows us to recover the thirdorder expansion for isotropic polycrystals of Willemse and Caspers. ${ }^{13}$

\section{APPENDIX C: CALCULATIONS FOR AGGREGATES OF ELLIPSOIDAL GRAINS}

We present an outline of the calculation of the tensors of the microgeometric parameters $A$ and $B$ for anisotropic aggregates of ellipsoids. It is convenient to consider first a single ellipsoid $G$ with the depolarization factors $L_{1}, L_{2}, L_{3}$ and the uniaxial or isotropic conductivity tensor $K_{G}$. We shall consider three different cases: (i) $k_{1}=z, k_{2}=k_{3}=1$; (ii) $k_{1}=k_{2}=z, k_{3}=1$; and (iii) $k_{1}=k_{2}=k_{3}=z$.

Case (i): $k_{1}=2, k_{2}=k_{3}=1$.

Denoting by $\mathrm{n}$ a unit vector in the axial ( $z$ ) direction, we have

$$
\mathrm{K}_{G}=I+(z-1) \mathrm{n} \oplus \mathrm{n},
$$

where $(\mathrm{n} \bullet \mathrm{n})_{i j}=n_{i} n_{j}$. We expand the corresponding single-grain polarizability $\mathscr{L}_{G}$ given by (28) in powers of $(z-1)$ to obtain

$$
\left.\frac{d^{2}}{d z^{2}} \mathscr{L}_{G}(z, 1,1)\right|_{z=1}=-2\left(\sum_{i=1}^{3} L_{i} n_{i}^{2}\right) \mathbf{n} \otimes \mathbf{n}
$$

and

$$
\left.\frac{d^{3}}{d z^{3}} \mathscr{L}_{G}(z, 1,1)\right|_{z=1}=6\left(\sum_{i=1}^{3} L_{i} n_{i}^{2}\right)^{2} \mathrm{n} \otimes \mathrm{n},
$$

where $n_{i}$ are the coordinates of $\mathbf{n}$ in a frame of principal axes of $\mathrm{G}$. The second and third derivatives of the average polarizability $\mathscr{L}$ of an array of ellipsoidal grains with semiaxes parallel to those of $G$ are obtained from (C2) and (C3) by averaging over the distributions of $\mathrm{n}$ and $L_{1}, L_{2}, L_{3}$. From (C1) the distribution of $n$ should be invariant under the reflections $n \rightarrow(-n)$. In particular, odd moments of $n_{i}$, $1 \leqslant i \leqslant 3$ vanish. We find that in the above reference frame, 


$$
\left.a \equiv \frac{d^{2}}{d z^{2}} \mathscr{L}_{G}^{p p}(z, 1,1)\right|_{z=1}=-2\left\langle\left(\sum_{i=1}^{3} L_{i} n_{i}^{2}\right) n_{p}^{2}\right\rangle
$$

and

$$
\left.c \equiv \frac{d^{3}}{d z^{3}} \mathscr{L}_{G}^{p p}(z, 1,1)\right|_{z=1}=6\left(\left(\sum_{i=1}^{3} L_{i} n_{i}^{2}\right)^{2} n_{p}^{2}\right\rangle,
$$

while the off-diagonal entries $\partial^{2} \mathscr{L}^{p q}, \partial^{3} \mathscr{L}^{p q}$ with $p \neq q$ are equal to zero.

Case (ii): $k_{1}=k_{2}=z, k_{3}=1$.

The conductivity within the grain is

$$
\mathrm{K}_{G}=1+(z-1)(\mathrm{I}-\mathbf{n} \otimes \mathbf{n}),
$$

where $\mathbf{n}$ is a unit vector in the axial direction. Substituting (C6) into (28) (with $\mathrm{O}=1$ ) and expanding in powers of $(z-1)$, we obtain formulas for $\partial^{2} / \mathscr{L}_{G}$ and $\partial^{3} / \mathscr{L}_{G}$, which, as in case (i), can be averaged over the distributions of $\mathbf{n}$ and L. In this way, we obtain the following formulas for the second and third derivatives of the average polarizability of an array of ellipsoids with parallel principal axes:

$$
\begin{aligned}
b & \left.\equiv \frac{d^{2}}{d z^{2}} \mathscr{L}^{p p}(z, z, 1)\right|_{z=1} \\
& =-\frac{2}{3}\left\langle L_{p}\right\rangle-2\left\langle\left(\sum_{i=1}^{3} L_{i} n_{i}^{2}\right) n_{p}^{2}\right\rangle
\end{aligned}
$$

and

$$
\begin{aligned}
d \equiv & \left.\frac{d^{3}}{d z^{3}} \mathscr{L}^{p p}(z, z, 1)\right|_{z=1}=6\left\langle\left(\sum_{i=1}^{3} L_{i}^{2} n_{i}^{2}\right) n_{p}^{2}\right\rangle \\
& +12\left\langle\left(\sum_{i=1}^{3} L_{i} n_{i}^{2}\right) L_{p} n_{p}^{2}\right\rangle-6\left\langle\left(\sum_{i=1}^{3} L_{i} n_{i}^{2}\right)^{2} n_{p}^{2}\right\rangle
\end{aligned}
$$

Case (iii): $k_{1}=k_{2}=k_{3}=z$.

In this case $\mathrm{K}_{G}=z \mid$ and using (28) and averaging, we obtain immediately

$$
\left.\frac{d^{2}}{d z^{2}} \mathscr{L}^{p p}(z, z, z)\right|_{z=1}=-2\left\langle L_{p}\right\rangle
$$

and

$\left.e \equiv \frac{d^{3}}{d z^{3}} \mathscr{L}^{p p}(z, z, z)\right|_{z=1}=6\left(L_{p}^{2}\right\rangle$.

We use formulas $(\mathrm{C} 4)$ through $(\mathrm{C} 10)$ to compute the partial derivatives of $\mathscr{L}\left(k_{1}, k_{2}, k_{3}\right)$ at $k_{i}=1$. Accordingly,

$$
\begin{aligned}
& \frac{\partial^{2}}{\partial k_{1}^{2}} \mathscr{L}^{p p}(1,1,1)=a, \\
& \frac{\partial^{2}}{\partial k_{1} \partial k_{2}} \mathscr{L}^{p p}(1,1,1)=\frac{1}{2}(b-2 a), \\
& \frac{\partial^{3}}{\partial k_{1}^{3}} \mathscr{L}^{p p}(1,1,1)=c, \\
& \frac{\partial^{3}}{\partial k_{1}^{2} \partial k_{2}} \mathscr{L}^{p p}(1,1,1)=\frac{1}{6}(d-2 c),
\end{aligned}
$$

and

$$
\frac{\partial^{3}}{\partial k_{1} \partial k_{2} \partial k_{3}} \mathscr{L}^{p p}(1,1,1)=\frac{1}{6}(e+3 c-3 d) \text {. }
$$

We substitute expressions ( $\mathrm{C} 11$ ) into the basic formulas (25) and (26) to obtain

$$
\mathrm{A}^{p p}=\frac{4}{3} a-\frac{1}{3} b=-2\left\langle\left(\sum_{i} L_{i} n_{i}^{2}\right) n_{p}^{2}\right\rangle+\frac{2}{9}\left\langle L_{p}\right\rangle
$$

and

$$
\begin{aligned}
\mathrm{B}^{p p}= & \frac{2}{3} c-\frac{2}{27} e-\mathrm{A}^{p p}=6\left\langle\left(\sum_{i=1}^{3} L_{i} n_{i}^{2}\right)^{2} n_{p}^{2}\right\rangle \\
& -4\left\langle\left(\sum_{I=1}^{3} L_{i} n_{i}^{2}\right) L_{p} N_{p}^{2}\right\rangle+\frac{4}{9}\left\langle L_{p}^{2}\right\rangle-\frac{2}{9}\left\langle L_{p}\right\rangle .
\end{aligned}
$$

Formulas $(\mathrm{Cl} 2)$ and $(\mathrm{C} 13)$ give the values of the eigenvalues of the average polarizability of an array of ellipsoids with parallel principal axes. The expressions for $A$ and $B$ for general configurations of ellipsoids can be obtained by replacing in $(\mathrm{C} 12)$ and $(\mathrm{C} 13)$ the tensor $\mathrm{L}$ by ${ }^{\circ} \mathrm{OLO}$ and averaging over the orientation field $O$. We restrict our attention to the case of uniformly distributed crystallographic orientations within each grain, which corresponds to $\mathbf{n}$ being uniformly distributed on the unit sphere in three-space. The relevant moments of the vector $\mathbf{n}$ are

$$
\left\langle n_{i}^{2}\right\rangle=\frac{1}{3}, \quad\left\langle n_{i}^{4}\right\rangle=\frac{1}{3}, \quad\left\langle n_{i}^{6}\right\rangle=\frac{1}{7}
$$

for $1 \leqslant i \leqslant 3$,

$$
\left\langle n_{i}^{2} n_{j}^{2}\right\rangle=\frac{1}{15}, \quad\left\langle n_{i}^{2} n_{j}^{4}\right\rangle=\frac{1}{35}
$$

for $i \neq j$, and

$$
\left\langle n_{1}^{2} n_{2}^{2} n_{3}^{2}\right\rangle=\frac{1}{105} \text {. }
$$

Substituting these values into $(\mathrm{C12})$ and $(\mathrm{C} 13)$, we obtain

$$
\begin{aligned}
\mathrm{A}^{p p}= & -\frac{2}{43}\left\langle L_{p}\right\rangle-\frac{2}{13} \\
\mathrm{~B}^{p p}= & \frac{32}{315}\left\langle L_{p}^{2}\right\rangle-\frac{2}{103}\left\langle L_{1}^{2}+L_{2}^{2}+L_{3}^{2}\right\rangle \\
& +\frac{2}{313}\left\langle L_{p}\right\rangle-\frac{4}{21},
\end{aligned}
$$

and $\mathrm{A}^{p q}=\mathrm{B}^{p q}=0$ for $p \neq q$. Replacing $\mathrm{L}$ by 'OLO and averaging over the grain orientation distribution yields expressions (32) and (33).

'Y. S. Touloukian, R. W. Powell, C. Y. Ho, and P. G. Klemens, Thermal Conductivity, I. Metallic Elements and Alloys; Thermal Conductivity II. Non-Metallic Solids, Thermodynamical Properties of Matter (IFI/Plenum, New York, 1970).

${ }^{2}$ W. Voigt, Lehrbuch der Kristallphysik (Teubner, Leipzig, 1928), p. 410. ${ }^{3}$ A. Reuss, Z. Angew. Math. Mech. 9, 49 (1929).

${ }^{4} \mathrm{O}$. Wiener, Abhandl, Math.-Phys. K1. Königl. Sachsischen Ges. 32, 509 (1912).

${ }^{5}$ M. Avellaneda, A. V. Cherkaev, K. A. Lurie, and G. W. Milton, J. Appl. Phys. 63, 4989 (1988).

${ }^{6}$ J. E. Molyneux, J. Math. Phys. 10, 912 (1969).

${ }^{7}$ J. E. Molyneux, J. Math. Phys. 11, 1172 (1970).

${ }^{8}$ P. B. Corson, J. Appl. Phys. 45, 3159 (1974).

${ }^{9}$ J. G. Berryman, J. Appl. Phys. 57, 2374 (1985).

${ }^{10}$ M. N. Miller, J. Math. Phys. 10, 1988 (1969). 
11Z. Hashin and S. Shtrikman, Phys. Rev، 130, 129 (1963).

${ }^{12}$ L. J. Walpole, J. Mech. Phys. Solids 14, 189 (1966).

${ }^{13}$ M. W. M. Willemse and W. J. Caspers, J. Math. Phys. 20, 1824 (1979).

${ }^{14} \mathrm{O}$. Bruno, "The effective conductivity of an infinitely interchangeable mixture", Ph. D. thesis, New York University, 1989.

${ }^{15}$ M. A. Elsayed, J. Math. Phys. 15, 2001 (1974).

${ }^{16} \mathrm{~J}$. C. Maxwell, Electricity and Magnetism (Clarendon, Oxford, 1873), 1st ed.; (see, for instance, J. A. Stratton, Electromagnetic Theory (McGrawHill, New York (1941), Chap. III.

${ }^{17}$ This is a consequence of the work in N. G. Meyers and A. Elcrat, Duke Math. J. 42, 121 (1975), on the $L^{\rho}$ integrability $(p>2)$ of gradients of solutions to elliptic equations. Considerations leading to estimate (80) can be found in M. Avellaneda, Commun. Pure Appl. Math. 40, 527 (1987), Proposition 8. 